\title{
Diagnóstico del desempeño docente en tiempos de pandemia en docentes del nivel inicial
}

\author{
Diagnosis of teacher performance in times of pandemic in initial level teachers
}

Diagnóstico do desempenho docente em tempos de pandemia em professores da pré-escola

Nancy Chambi Condori

nchambi@unap.edu.pe

https://orcid.org/0000-0002-4520-4707

Universidad Nacional del Altiplano, Puno-Perú
Nelly Olga Zela Payi

nzela@unap.edu.pe

https://orcid.org/0000-0001-9119-1102

Universidad Nacional del Altiplano, Puno-Perú

Artículo recibido 03 de septiembre 2021, arbitrado y aceptado 06 de octubre 2021 y publicado 13 de diciembre 2021

\section{RESUMEN}

La presente investigación se realizó con el objetivo de: Determinar el desempeño docente en tiempos de pandemia en docentes del nivel Inicial del ámbito de la UGEL Puno. La investigación fue de tipo descriptivo transversal con diseño no experimental. La población de estudio estuvo conformada por el total de 180 docentes del nivel Inicial del ámbito de la UGEL Puno. Para recolectar los datos se utilizó la técnica de la Encuesta y como instrumento un Cuestionario compartido por Google form, de acuerdo a la estadística descriptiva porcentual se obtuvo los siguientes resultados: El 65.56\% de los docentes de educación básica regular en tiempos de pandemia tienen un desempeño regular, el $25.56 \%$ deficiente y un $8.89 \%$ su desempeño bueno. Referente a la práctica de la comunicación institucional predomina el desempeño regular con un $76.67 \%$, así mismo en el $72.22 \%$ sobre el uso de llamadas telefónicas, $68.33 \%$ en interacción directa virtual, $77.78 \%$ remiten hojas de trabajo, $74.44 \%$ dominio de classrooom, $87.78 \%$ en la comprensión didáctica con envío de videos y el $65.56 \%$ en el ejercicio compartido por WhatsApp. Visto los resultados se concluye que los docentes del nivel Inicial poseen en forma global un desempeño regular, así como en cada una de sus dimensiones.

Palabras clave: Desempeño Docente; Tiempos de pandemia; Nivel Inicial

\section{ABSTRACT}

This research was carried out with the objective of: Determining the teaching performance in times of pandemic in teachers of the Initial level of the UGEL Puno area. The research was of a descriptive cross-sectional type with a nonexperimental design. The study population was made up of a total of 180 teachers of the Initial level of the UGEL Puno area. To collect the data, the Survey technique was used and as an instrument a Questionnaire shared by Google form, according to the percentage descriptive statistics, the following results were obtained: $65.56 \%$ of Regular basic education teachers in times of pandemic have a regular performance, $25.56 \%$ poor and $8.89 \%$ good performance. Regarding the practice of Institutional communication, regular performance predominates with $76.67 \%$, likewise in $72.22 \%$ on the use of telephone calls, $68.33 \%$ in direct virtual interaction, $77.78 \%$ send worksheets, $74.44 \%$ domain of classroom, $87.78 \%$ in didactic comprehension with sending videos and $65.56 \%$ in the exercise shared by WhatsApp. Considering the results, it is concluded that the teachers of the Initial level have a regular performance globally, as well as in each of its dimensions.

Key words: Teaching Performance; Pandemic Times

\section{RESUMO}

Esta pesquisa foi realizada com o objetivo de: Determinar o desempenho docente em tempos de pandemia em professores do nível Inicial da UGEL Puno. A pesquisa foi do tipo transversal descritiva com desenho não experimental. A população do estudo foi constituída por um total de 180 professores do nível Inicial da UGEL Puno área. Para a coleta de dados, utilizouse a técnica Survey e como instrumento um Questionário compartilhado pelo formulário Google, de acordo com a estatística descritiva percentual, foram obtidos os seguintes resultados: $65,56 \%$ dos professores regulares da educação básica em tempos de pandemia têm desempenho regular, $25,56 \%$ ruim e $8,89 \%$ bom desempenho. Em relação à prática de comunicação institucional, o desempenho regular predomina com $76,67 \%$, assim como em $72,22 \%$ no uso de ligações telefônicas, $68,33 \%$ na interação virtual direta, $77,78 \%$ enviam planilhas, $74,44 \%$ domínio de clasrrom, 87,78\% na compreensão didática com envio vídeos e $65,56 \%$ no exercício compartilhado pelo WhatsApp. Considerando os resultados, conclui-se que os professores do nível Inicial têm um desempenho regular globalmente, bem como em cada uma de suas dimensões.

Palavras-chave: Desempenho de Ensino; Tempos de pandemia; Nível iniciante 


\section{INTRODUCCIÓN}

El desempeño profesional en Calidad en salud contiene en su definición el cumplimiento de las funciones de los profesionales de las Ciencias Médicas donde la ética y el comportamiento humano resultan esenciales. Se considera que esta categoría socio-pedagógica, tiene cinco dimensiones: político- legal, ético-motivacional, técnico-profesional, científico-tecnológica y gestión (Mejía Sánchez y Borgues Oquendo, 2021).

En el estudio se demostró que existe relación significativa entre desempeño docente y el rendimiento académico de los estudiantes de las promociones 2011 y 2012 del ciclo académico 2015 I en la Facultad de Tecnología (Guisado Salazar et al., 2020).

De acuerdo a los resultados se ha podido establecer que es eficaz el uso de las rúbricas, dado que su carácter descriptivo ayuda a identificar mejor los aspectos evaluados, pudiendo establecerse niveles de logro en base a una escala. De acuerdo a los resultados estadísticos que corroboran los datos obtenidos en el trabajo de campo se aprecia y existe un nivel de significancia (Sig.), 0,000 alfa, con un valor de Prueba de Correlación de Pearson 0,532** para un número de 76 casos, para la determinación de la correlación entre uso de la rúbrica como herramienta de evaluación formativa y sumativa y calidad del desempeño docente en las Instituciones Educativas de primaria con enfoque intercultural (Arévalo Quijano et al., 2020).

La gestión del clima institucional constituye un elemento importante en el desempeño del personal docente en la excelencia de su trabajo, y por tanto, en la motivación y aprendizaje de sus estudiantes. Para cumplir su cometido, institutos y universidades requieren ponerse a tono con las exigencias sociales del tiempo histórico en el cual se desenvuelven, y en consonancia, con cada tiempo concreto, modificar, de manera sustancial si es preciso, los basamentos y concepciones sobre las que sustentan sus procesos formativos y su dinámica de labor. Para conseguirlo, especial atención reclama el papel del directivo y su capacidad de liderazgo en el desempeño del personal docente en cuanto a las capacidades pedagógicas, la responsabilidad en el desempeño de sus funciones, las relaciones interpersonales y los resultados de su labor educativa (More Espinoza y Morey Guevara, 2021).

En relación con la dimensión factores del desempeño laboral se puede concluir que factores tales como conocimiento del trabajo, producción, responsabilidad, capacidad de liderazgo e identidad laboral son para los directivos de uso frecuente en la práctica educativa en la Institución Universitaria ITSA. A pesar de que los docentes declaran una frecuencia regular con respecto a estos factores, se observa que debería aumentar su aplicación en la experiencia docente de los maestros encuestados (Rodríguez-Marulanda y Lechuga-Cardozo, 2019).

Los factores afectivos juegan un papel preponderante en el desempeño docente y los profesores mejoraron de manera significativa. En primer lugar, pudimos identificar que los profesores de educación básica se sentían desmotivados al momento de llevar a cabo su labor docente. De acuerdo a la prueba actitudinal, se pudo corroborar dicha información, pues lo docentes no sentían satisfacción por su trabajo. En segundo lugar, pudimos detectar que con el aumento de incentivos motivacionales externos el desempeño del profesor de educación básica adoptó modificaciones y el 
resultado se observa en la segunda evaluación de carácter académico. En tercer lugar, a partir de Diseño Instruccional motivacional externo se pudo analizar la motivación en los profesores de educación básica. En adición, la pertinencia del trabajo dependerá de las instituciones, pues se requiere de disponibilidad por parte de directivos para que se pueda llevar a cabo. En cuanto a la viabilidad del Diseño Instruccional se considera eficaz para aplicarlo en diversos momentos. Esto para poder cubrir las necesidades, al tiempo de solicitar apoyo del personal del departamento psicopedagógico para su auxilio. Las limitaciones son, precisamente el no contar con un departamento que pueda brindar apoyo con respecto a los talleres y su aplicación (Gallardo Bonilla y Pacheco Sosa, 2019).

El contar con una evaluación continua del desempeño docente desde la perspectiva de la práctica profesional permite tener evidencia real y contextualizada de las situaciones que enfrenta el profesor en su actuar diario durante diversos momentos del ciclo escolar, por lo tanto, se detectan las áreas de oportunidad de cada maestro, de la escuela e incluso de la zona escolar, lo cual se convierte en un insumo para desarrollar un plan para abatir esto, con el fin de que repercuta en el desempeño profesional y por extensión en el proceso de aprendizaje del alumno (Martínez Chairez et al., 2020).

El problema destacado por los profesores investigados y el análisis realizado señala la contradicción presente en el contexto de una política educativa pública importante para el proceso de PDD. Esto se debe a que, en opinión de los profesores, el DAD aún no ha producido un impacto significativo para la mejora del proceso de enseñanza y aprendizaje, en la medida en que no colabora con la formación del profesorado de forma coherente y eficaz (Lima Jardilino et al., 2021).

Se relacionó el clima organizacional con las dimensiones del desempeño laboral, cuyo resultado manifiesta una mayor correlación con la dimensión emocionalidad, donde $r=, 950$ es decir 92\%, mientras que la de menor grado fue las relaciones interpersonales, donde $r=, 744$ con $55 \%$, pues se percibe que los colaboradores se sienten satisfechos, con sentimientos favorables debido a que sus niveles de emociones son positivos; sin embargo, son poco solidarios entre compañeros de trabajo (Verde Gonzales, 2021).

La relación entre el monitoreo docente y las prácticas pedagógicas en la Institución Educativa La Merced de Lima de San Juan de Miraflores 2020, a un nivel alto con un $61,9 \%$. Asimismo, posee 0,811 de coeficiente de correlación y su significancia $\mathrm{p}$-valor $=0,000<0.05$, por ello se concluye que hay una relación significativa entre competencias monitoreo docente y las practicas pedagógicas (Ruiz acuña, 2020).

Se ha logrado definir que existe relación significativa entre las variables de estudio, inteligencia emocional y desempeño docente en las clases virtuales de los profesores de la Red 22, los olivos, 2020. El valor del coeficiente de correlación Rho de Spearman 0,811 nos indica que existe una correlación positiva alta (Quispe Malaver, 2020).

A través de las capacitaciones que se brindó con los talleres utilizando herramientas tecnológicas se logró que los docentes a quienes se impartieron estos programas mejoren su desempeño docente con la adquisición de nuevos conocimientos útiles 
y que beneficiaran ya que son utilizados en estos tiempos de pandemia y que de esta manera pueden llegar a sus estudiantes de una forma más didáctica, creativa y significativa (Herrera Garzón, 2021).

Se estableció que entre las variables Acompañamiento pedagógico y la variable Desempeño docente existe una relación significativa con un p_valor $=0.002<0.05$. El coeficiente rho de Spearman $=0.330$ corresponde a una relación positiva baja, por otra parte, se determinó que entre la dimensión Curso y tutoriales virtuales y la variable Desempeño docente existe una relación significativa con un p_valor $=0.034<0.05$. El coeficiente rho de Spearman $=0.235$ corresponde a una relación positiva baja (Meza Castañeda, 2021).

Concluye que existe incidencia significativa de las competencias digitales sobre el desempeño de los docentes. Esta conclusión es corroborada con el valor p igual a 0,000; que es menor a 0,005. Asimismo, el coeficiente de Nagelkerke 0,689 indica que existe incidencia de la variable independiente sobre la variable dependiente (Maguiña Polanco, 2021).

Se han podido analizar las características de un curso de capacitación docente y se han detectado las necesidades de formación. Los resultados de este trabajo de investigación desvelaron una necesidad de actualización en saberes tecno pedagógicos por parte del personal académico del bachillerato mexicano. Se constató que, a inicios de la pandemia, existía una necesidad imperante de capacitación. Se demostró que hubo avance en el desarrollo de competencias a partir del curso ofertado. Sin embargo, es cierto que los procesos de formación no se limitan a un curso virtual, sino que requieren de una oferta articulada y sistematizada que garantice la continuidad del desarrollo de competencias acordes a los intereses y a las necesidades del profesorado (González Fernández, 2021).

La dimensión pedagógica es vital en todo docente, en relación con despertar en los estudiantes el interés por aprender, así como la puesta de familiaridad en sus medios para el logro de las capacidades que se requieren adquirir, a pesar de las dificultades que pudieran suscitarse en cualquier contexto socioeconómico y cultural. En relación a esta se concluye que los docentes despiertan el interés en el desarrollo de las experiencias de aprendizaje y que los estudiantes aprenden mejor en la estrategia aprendo en casa con los siguientes recursos educativos didácticos: Videos, audios, fichas técnicas, imágenes, televisión, textos (Farfán Chávez, 2021).

El estrés laboral se relaciona de manera inversa, baja y significativa con el desempeño docente (Rho - ,233 y p-valor ,001). Es decir que a mayor estrés laboral va existir un mal desempeño docente, por otra parte el cansancio emocional se relaciona de manera inversa, baja y significativa con el desempeño docente (Rho - ,344 y p-valor ,003). Es decir, que a un mayor cansancio emocional va a ver un mal desempeño docente (Collantes Cieza, 2021).

El Plan de fortalecimiento de capacidades dirigido a docentes contiene estrategias de capacitación a través de talleres, webinars sobre estrategias didácticas innovadoras para la enseñanza remota-virtual, uso eficiente de recursos tecnológicos educativos y mejora del clima laboral. Los conocimientos obtenidos fueron usados por los docentes mejorando el proceso de enseñanza aprendizaje (Abanto Perez, 2021). 
Se determinaron niveles medios y altos de actitud hacia el uso de las TICs en docentes de Lima en confinamiento por COVID-19, relacionado a la disponibilidad y conocimiento de estas, así mismo se determinó una baja percepción de desempeño en docentes de Lima en confinamiento por COVID-19, relacionado a las limitaciones que se presentaron debido al cambio del método de enseñanza (Choquecota Quinta y Quispe Livias, 2021).

En la actualidad en el campo de la Educación surgió un cambio radical en el desarrollo de la labor educativa, en tiempos de pandemia por la COVID 19, Según UNESCO (2020), mil doscientos millones de estudiantes han sido perjudicados por el cierre de sus escuelas y universidades. Al mismo tiempo, la migración de todo el proceso de enseñanza aprendizaje a un entorno virtual trajo consigo algunas interrogantes respecto a los docentes y a su capacidad para desenvolverse adecuadamente. En el contexto universitario Peruano, se ha desarrollado una migración gradual a la virtualidad, donde muchas casas de estudio han tenido que agenciarse de los recursos en línea necesarios para el inicio de las actividades académicas. Si bien este proceso de adaptación es temporal hasta el eventual regreso a las aulas, es una oportunidad enorme para repensar el proceso de enseñanza aprendizaje y evaluar todas las dimensiones del desempeño docente. Por lo que, el desempeño docente debe ir a la par en referencia a los avances del desarrollo educativa (Márquez, 2017), el rol del docente en tiempos de pandemia se adecúa al cambio y adaptarse no fue nada sencillo pero tampoco imposible, asumieron responsabilidades para interactuar con los estudiantes la que ayudó a mejorar la práctica docente, (Guaman, 2020). Las docentes del nivel inicial en este nuevo contexto deben tener predisposición a cambiar los modelos tradicionales y encontrar roles más participativos, colaborativos para que esta situación que nos trajo la pandemia se traduzca en un cambio educativo, (Hurtado, 2020). Por otro lado los resultados de la pandemia en la educación actual se ha visto afectada de forma radical por la emergencia de una cultura digital, provocando en los docentes y estudiantes la imperiosa necesidad de conocer y adaptarse a ese cambio (Tejedor et al., 2020). El desempeño docente son las actuaciones observables de la persona que pueden ser descritas, evaluadas y que expresan su competencia, bajo tres condiciones: actuación observable en correspondencia a una responsabilidad y logro de determinados resultados (MINEDU, 2016). Esta situación como lo refiere Pérez (2006), permitirá establecer una comunicación, donde es necesario el reconocimiento del otro, ya que de esta manera se produce la comunicación, donde los protagonistas imponen su código a otro de dos maneras; o bien es un proceso asimilador que niega la identidad del otro, o bien es un proceso de indiferencia o marginador.

En definitiva esta competencia regular es señal que el docente no está capacitado ni habilitado para la interacción virtual ni de las funciones académicas que les compete" (Martínez et al., 2006:2). Este hecho en el desempeño del docente del nivel inicial no permitirá recuperar la matriz propia que, aunque difusa, es el punto de partida para acompañar procesos de construcción y fortalecimiento de identidades (Montilla, 2010). En las tecnologías de información y comunicación, adecuarse al cambio y adaptarse no fue nada sencillo pero tampoco imposible, asumieron responsabilidades 
con desaprender lo que no nos sirve y aprender lo que ayudó a mejorar la práctica docente, (Guaman, 2020), no obstante todos los países a pesar de no contar con una estrategia nacional de educación remota o a distancia consolidada, menos para una emergencia, han experimentado atrevimientos significativo (Álvarez et al., 2020).

\section{MÉTODO}

La metodología que se aplicó fue de tipo descriptivo transversal con diseño no experimental. Cuya población estuvo conformada por un total de 180 docentes del nivel Inicial del ámbito de la UGEL Puno. La información se obtuvo con la aplicación de un cuestionario propuesto y diseñado por medio del formulario de Google Form, fundamentada, en conocer su rol como docente del nivel inicial, a fin de ver los resultados del desempeño docente en tiempos de pandemia, aquella que nace en la interrelación con los estudiantes, la que se realiza simultáneamenteenlarecolecciónyel procesamiento de los datos. En esta metodología, las investigadoras se encuentran inmerso en el campo de estudio y junto a las respectivas dimensiones, con la finalidad de fortalecer la comprensión del fenómeno social investigado, (Bonilla y López, 2016), la elaboración del cuestionario fue semiestructurada y sujeta a las dimensiones relacionadas con los objetivos del estudio en una matriz apriorística, en la cual se ha distinguido dichas dimensiones del campo de estudio las que son significativas desde la propia investigación. (Cisterna, 2005).

\section{RESULTADOS Y DISCUSIÓN}

En la actualidad diversos países promueven, éste propósito, del marco del Buen desempeño Docente para la Buena Enseñanza, y la UGEL
Puno, no es exenta a éste propósito y más aún en tiempos de pandemia por la COVID 19. El fortalecimiento y construcción del Diagnóstico del Desempeño Docente en maestras del nivel inicial es principalmente un ejercicio de reflexión sobre el sentido de esta profesión y su función en la sociedad, la cultura y el desarrollo con equidad. Constituye una interrelación entre sujetos (Docente-niños), y se aspira a movilizar el logro de un desarrollo profesional permanente la que garantiza estos aprendizajes. Se trata por ser la enseñanza y el quehacer de los docentes en Tiempos de pandemia, la que exige responsabilidad en las dimensiones de la práctica de la comunicación Institucional, el uso de llamadas telefónicas, la interacción directa de manera virtual, la remisión de hojas de trabajo, el dominio del classrom, la comprensión didáctica con envío de videos, el ejercicio compartido por WhatsApp. Las que en la actualidad son modalidades del trabajo remoto virtual, y que aún requieren fortalecer sus competencias digitales y responder de manera adecuada la implementación de una auténtica educación a distancia, siendo como sigue:

Los resultados del desempeño docente en tiempos de pandemia del COVID 19, se observa que el $65.56 \%$ de docentes tienen un desempeño regular en tanto un $25.56 \%$ desempeño deficiente y el $8.89 \%$ desempeño bueno. De acuerdo a los resultados podemos inferir que aún se tiene poco conocimiento para el desarrollo del proceso educativo en su plenitud. (Tabla 1). 
Tabla 1. Diagnóstico del desempeño docente en tiempos de pandemia en docentes del nivel inicial.

\begin{tabular}{ccc}
\hline DESEMPENO DOCENTE & $\mathbf{N}^{\circ}$ & $\%$ \\
\hline Bueno & 16 & 8.89 \\
Regular & 118 & 65.56 \\
Deficiente & 46 & 25.56 \\
\hline TOTAL & $\mathbf{1 8 0}$ & $\mathbf{1 0 0 . 0 0}$ \\
\hline
\end{tabular}

Fuente: Cuestionario desempeño docente en tiempos de pandemia.

Alanalizar los resultados del desempeño docente en la dimensión de comunicación Institucional, se evidencia que el $76.67 \%$ de docentes tienen un desempeño regular seguido por $13.33 \%$ bueno y el $10.00 \%$ muestra un desempeño deficiente. Estos resultados evidencian que el desempeño en cuanto a la comunicación Institucional es eminentemente regular. (Tabla 2).

Tabla 2. Diagnóstico del desempeño docente: práctica de comunicación institucional.

\begin{tabular}{ccc}
\hline COMUNICACIÓN INSTITUCIONAL & $\mathbf{N}^{\circ}$ & $\mathbf{\%}^{\circ}$ \\
\hline Bueno & 24 & 13.33 \\
Regular & 138 & 76.67 \\
Deficiente & 18 & 10.00 \\
\hline TOTAL & $\mathbf{1 8 0}$ & $\mathbf{1 0 0 . 0 0}$ \\
\hline
\end{tabular}

Fuente: Cuestionario desempeño docente en tiempos de pandemia.

De acuerdo a los resultados obtenidos en la presente Tabla 3 muestran que el $72.22 \%$ de las docentes del nivel inicial en la dimensión llamadas telefónicas, tienen un desempeño regular en tanto un $17.78 \%$ bueno y un $10.00 \%$ deficiente, resultados que demuestran que la mayoría de los docentes aplican las llamadas telefónicas durante la pandemia.

Tabla 3. Diagnóstico del desempeño docente: uso de llamadas telefónicas.

\begin{tabular}{ccc}
\hline USO DE LLAMADAS TELEFÓNICAS & $\mathbf{N}^{\circ}$ & $\%$ \\
\hline Bueno & 32 & 17.78 \\
Regular & 130 & 72.22 \\
Deficiente & 18 & 10.00 \\
\hline TOTAL & $\mathbf{1 8 0}$ & $\mathbf{1 0 0 . 0 0}$ \\
\hline
\end{tabular}

Fuente: Cuestionario desempeño docente en tiempos de pandemia.

Los resultados demuestran que en la Tabla 4 evidencian que el desempeño del docente sobre la interacción directa virtual es regular en el 68.33\%, solo un $22.22 \%$ muestra un desempeño bueno y el $9.44 \%$ un desempeño deficiente. Resultados que demuestran que los docentes presentan imprecisiones sobre la interacción directa virtual. 
Tabla 4. Diagnóstico del desempeño docente: interacción directa virtual.

\begin{tabular}{ccc}
\hline INTERACCIÓN DIRECTA VIRTUAL & $\mathbf{N}^{\circ}$ & $\mathbf{\%}$ \\
\hline Bueno & 40 & 22.22 \\
Regular & 123 & 68.33 \\
Deficiente & 17 & 9.44 \\
\hline TOTAL & $\mathbf{1 8 0}$ & $\mathbf{1 0 0 . 0 0}$ \\
\hline
\end{tabular}

Fuente: Cuestionario desempeño docente en tiempos de pandemia.

En los resultados de la presente Tabla 5 se destacan el desempeño docente respecto al dominio de clasrrom, donde se evidencia que el $74.44 \%$ tiene un desempeño regular seguido por $15.56 \%$ desempeño bueno y el $10.00 \%$ es deficiente. Estos resultados demostraron que el desempeño aún falta por capacitar esta dimensión ya que predomina de forma regular.

Tabla 5. Diagnóstico del desempeño docente: dominio de clasroom.

\begin{tabular}{ccc}
\hline DOMINIO DE CLASRROM & $\mathbf{N}^{\circ}$ & \% \\
\hline Bueno & 28 & 15.56 \\
Regular & 134 & 74.44 \\
Deficiente & 18 & 10.00 \\
\hline TOTAL & $\mathbf{1 8 0}$ & $\mathbf{1 0 0 . 0 0}$ \\
\hline
\end{tabular}

Fuente: Cuestionario desempeño docente en tiempos de pandemia.

Dados los resultados sobre el desempeño docente respecto a la comprensión didáctica con envío de videos, en la Tabla 6 se muestra que, el $87.78 \%$ muestra un desempeño regular, el $8.89 \%$ desempeño deficiente y solo un $3.33 \%$ desempeño bueno; esto demuestra que los docentes aún no están capacitados para comprender la dimensión que involucra la edición de videos.

Tabla 6. Diagnóstico del desempeño docente: comprensión didáctica con envío de videos.

\begin{tabular}{ccc}
\hline COMPRENSIÓN DIDÁCTICA CON ENVÍO DE VIDEOS & $\mathbf{N}^{\circ}$ & $\%$ \\
\hline Bueno & 6 & 3.33 \\
Regular & 158 & 87.78 \\
Deficiente & 16 & 8.89 \\
\hline TOTAL & $\mathbf{1 8 0}$ & $\mathbf{1 0 0 . 0 0}$ \\
\hline
\end{tabular}

Fuente: Cuestionario desempeño docente en tiempos de pandemia.

Los resultados del desempeño docente sobre el uso de WhatsApp que se muestran en la Tabla 7, donde se observa que, el $65.56 \%$ de los docentes tienen un desempeño regular, un $25.56 \%$ desempeño deficiente y el $8.89 \%$ desempeño bueno. Con estos resultados podemos inferir que el uso del WhatsApp no se aplica en su plenitud en tiempos de pandemia. 
Tabla 7. Evaluar el desempeño docente: uso de whatsapp

\begin{tabular}{ccc}
\hline USO DE WHATSAPP & $\mathbf{N}^{\circ}$ & $\%$ \\
\hline Bueno & 16 & 8.89 \\
Regular & 118 & 65.56 \\
Deficiente & 46 & 25.56 \\
TOTAL & $\mathbf{1 8 0}$ & $\mathbf{1 0 0 . 0 0}$ \\
\hline
\end{tabular}

Fuente: Cuestionario desempeño docente en tiempos de pandemia.

\section{Discusión}

El desempeño docente, implica asumir un reto en esta nueva modalidad de educación no presencial. De acuerdo a los resultados obtenidos se tiene que El 65.56\% de los docentes de educación básica Regular en tiempos de pandemia tienen un desempeño regular, el $25.56 \%$ deficiente y un $8.89 \%$ su desempeño bueno. Por lo que, el desempeño docente debe ir a la par en referencia a los avances del desarrollo educativa (Márquez, 2017). es así que la práctica de la comunicación Institucional predomina el desempeño regular con un $76.67 \%$, ya que el rol del docente en tiempos de pandemia se adecúa al cambio y adaptarse no fue nada sencillo pero tampoco imposible, asumieron responsabilidades para interactuar con los estudiantes la que ayudó a mejorar la práctica docente, (Guaman, 2020).

Las docentes del nivel inicial en este nuevo contexto deben tener predisposición a cambiar los modelos tradicionales y encontrar roles más participativos, colaborativos para que esta situación que nos trajo la pandemia se traduzca en un cambio educativo, (Hurtado, 2020). Por otro lado los resultados de la pandemia en la educación actual se ha visto afectada de forma radical por la emergencia de una cultura digital, provocando en los docentes y estudiantes la imperiosa necesidad de conocer y adaptarse a ese cambio (Tejedor et al., 2020).
También es importante mencionar que el desempeño docente, según el estudio de Estrada y Mamani, determinó que existe una correlación significativa entre el desempeño del docente y el compromiso organizacional. Así mismo, la investigación de Kú-Hernández y Pool-Cibrián tiene por conclusión que no se encontraron relaciones significativas entre el desempeño docente y variables relativas a los docentes (procedencia, edad, género). Entre otros aspectos, y existe una constante necesidad de fomentar una cultura de evaluación al docente. Es necesario que el docente comprenda que la evaluación de su desempeño es un medio de retroalimentación que lo llevará a fortalecer dimensiones de su desempeño que requieran mejoras.

$\mathrm{Al}$ respecto, en el estudio se ha encontrado que el desempeño es eminentemente regular. Estos hallazgos demuestran que las docentes del nivel Inicial no están debidamente preparados, pues un nivel regular refleja deficiencia en algunos aspectos del desarrollo de interacción las que determinan inconsistencia en su desempeño.

$\mathrm{Al}$ evaluar el desempeño docente mediante la dimensión práctica dela comunicación institucional, predomina también el desempeño regular. Estos resultados evidencian que el desempeño en cuanto a la comunicación institucional es eminentemente regular. El desempeño docente son las actuaciones 
observables de la persona que pueden ser descritas, evaluadas y que expresan su competencia, bajo tres condiciones: actuación observable en correspondencia a una responsabilidad y logro de determinados resultados (MINEDU, 2016). Esta situación como lo refiere Pérez (2006), permitirá establecer una comunicación, donde es necesario el reconocimiento del otro, ya que de esta manera se produce la comunicación, donde los protagonistas imponen su código a otro de dos maneras; o bien es un proceso asimilador que niega la identidad del otro, o bien es un proceso de indiferencia o marginador.

Respecto al desempeño docente sobre el uso de llamadas telefónicas, los resultados muestran que se tiene un desempeño regular en la mayoría de los docentes (cuadro 3) lo que significa que se está aplicando en forma regular las llamadas telefónicas durante el proceso educativo. Con estos resultados se puede inferir que el desempeño regular en Docentes del nivel inicial, lo que constituye una limitante para interactuar con los estudiantes.

En relación a la dimensión interacción directa virtual, también predomina un desempeño regular, aunque en este aspecto una quinta parte de los docentes tienen un buen desempeño. La exposición de estos resultados demuestra que la mayoría de los docentes presentan imprecisiones en la interacción directa virtual. En definitiva esta competencia regular es señal que el docente no está capacitado ni habilitado para la interacción virtual ni de las funciones académicas que les compete" (Martínez et al., 2006:2).

Sobre la dimensión remiten hojas de trabajo, los resultados también muestran un desempeño regular, y el desempeño solo se da en la décima parte de los docentes. Este hecho en el desempeño del docente del nivel inicial no permitirá recuperar la matriz propia que, aunque difusa, es el punto de partida para acompañar procesos de construcción $\mathrm{y}$ fortalecimiento de identidades (Montilla, 2010, p 36).

En tanto, sobre el desempeño dominio de clasrrom, predomina el desempeño regular y el desempeño bueno en minina proporción. El rol del docente debe apoyarse en las tecnologías de información y comunicación, adecuarse al cambio y adaptarse no fue nada sencillo pero tampoco imposible, asumieron responsabilidades con desaprender lo que no nos sirve y aprender lo que ayudó a mejorar la práctica docente, (Guaman, 2020).

Los resultados del desempeño docente sobre el uso del WhatsApp, se muestran en los resultados, es regular a deficiente dado que la cuarta parte de los docentes presenta desempeño deficiente. Con estos resultados podemos inferir que no se aplica en su plenitud, por problemas de conectividad. No obstante todos los países a pesar de no contar con una estrategia nacional de educación remota o a distancia consolidada, menos para una emergencia, han experimentado atrevimientos significativo (Álvarez et al., 2020).

\section{CONCLUSIONES}

De un total de 180 docentes del nivel inicial del ámbito de la UGEL Puno, evaluados que representa al $100 \%$, la mayoría (87.78\%) posee desempeño regular, el desempeño bueno se encuentra en menos de la décima (7.78\%) parte y el desempeño deficiente en mínima proporción (4.44\%); resultados que determinan que el docente no está debidamente 
preparado, pues un nivel regular refleja deficiencia en muchos aspectos que determinan inconsistencia en su desempeño. Es importante mencionar que las docentes del nivel inicial del ámbito de la UGEL Puno, en estos tiempos de pandemia COVID 19, vienen enfrentando momentos complejos de trabajo, por el cambio drástico de la educación presencial a una educación no presencial o virtual, en ese contexto vienen a travesando momentos difíciles a una serie de retos y desafíos, como la de conceptuar e implementar la educación remota, es así que el desempeño docente en estos tiempos de pandemia está se tiene dificultades en la interacción con los estudiantes y aún no se viene implementando capacitaciones permanentes de parte del MED. Si bien existen estudios que demuestran una rápida adaptación a contextos no presenciales en otros contextos, en el Perú la transición fue más lenta y una de las posibles razones es el retraso es la habilitación de plataformas virtuales y otros medios que viabilicen esta migración. Cabe decir que, en otros contextos diferentes al Perú, se ha demostrado que uno de los factores más importantes que sirvió de soporte al desempeño de los docentes en tiempos de pandemia fue la asistencia institucional en el desarrollo de las sesiones de enseñanza.

\section{REFERENCIAS}

Abanto Perez, F. A. (2021). Condiciones laborales que afectan eldesempeñolaboral en la Institución Educativa Particular Anglo Americano, 2019 [Universidad César Vallejo]. In Universidad César Vallejo. https://repositorio.ucv.edu.pe/ bitstream/handle/20.500.12692/52048/Jaimes_ VCM-SD.pdf? sequence $=1$ \&isAllowed $=y$

Álvarez, H., Arias, E., Bergamaschi, A., López, A., Ortiz, M., Pérez, M., Rieble, S., Rivera, M., Scannone, R., Vásquez, M. y Vieteri, A. (2020). La educación remota en tiempos del coronavirus, los sistemas educativos de América Latina y el Caribe ante COVID-19. BID. Mayo 2021. https://publications.iadb.org/publications/ spanish/document/La-educacion-en-tiemposdel-coronavirus-Los-sistemas-educativos-deAmerica-Latina-y-el-Caribe-ante-COVID-19. pdf

Arévalo Quijano, J. C., Castro Paniagua, W. G., y Leguía Carrasco, Z. J. (2020). The rubric as an evaluation instrument and teaching per- formance with intercultural approach in primary educa- tional institutions in Peru. Revista Conrado, 16(73), 14-20. http://scielo.sld. $\mathrm{cu} /$ scielo.php? script $=$ sci_abstract\&pid $=$ S1990$86442020000200014 \& \operatorname{lng}=$ es\&nrm $=$ iso

Bonilla, M. Á. y López, A. D. (2016). Ejemplificación del proceso metodológico de la teoría a fundamentada. Cinta de Moebio (57), 305-315. https://www.moebio.uchile.cl/57/bonilla.html

Choquecota Quinta, K., y Quispe Livias, E. J. (2021). Actitud hacia el uso de las TICS y desempeño autopercibido en docentes de Lima en confinamiento por Covid-19 [Universidad San Ignacio de Loyola]. In Universidad San Ignacio de Loyola. http://repositorio.usil.edu.pe/ handle/USIL/11254\%0Ahttp://repositorio.usil. edu.pe/handle/USIL/11254

Cisterna, F. (2005). Categorización y triangulación como procesos de validación del conocimiento en investigación cualitativa. Theoria, 14(1), 61-71. https://www.redalyc.org/articulo. oa?id=29900107

Collantes Cieza, Y. (2021). Estrés laboral y desempeño docente en la Institución Educativa $\mathrm{N}^{\circ} 52005$ Nuestra Señora de las Mercedes, Madre de Dios, 2021 [Universidad César Vallejo]. In Universidad César Vallejo (Issue October 2013). http://repositorio.uncp.edu.pe/ bitstream/handle/UNCP/3000/Silva Acosta. pdf ? sequence $=1$ \&is Allowed $=y \% 0$ Ahttps: $/ /$ repositorio.comillas.edu/x mlui/ handle/11531/1046

Farfán Chávez, K. L. (2021). Evaluación del Desempeño Docente en la Estrategia" Aprendo en Casa" de los Estudiantes del Nivel Secundaria 
delaI.EN¹4739 Paita-2020 [Universidad César Vallejo]. In Universidad César Vallejo (Issue October 2013). http://repositorio.uncp.edu.pe/ bitstream/handle/UNCP/3000/Silva Acosta. pdf? sequence $=1$ \&isAllowed $=y \% 0$ Ahttps: $/ /$ re positorio.comillas.edu/x mlui / handle/11531/1046

Gallardo Bonilla, M. A., y Pacheco Sosa, C. (2019). Evaluación Docente Y Desempeño Profesional: En Juego La Condición Afectiva Del Profesorado. Revista de La Escuela de Ciencias de La Educación, 2(14), 81-89. https:// doi.org/10.35305/rece.v2i14.440

González Fernández, M. O. (2021). La capacitación docente para una educación remota de emergencia por la pandemia de la COVID-19. Revista Tecnología, Ciencia y Educación, 19(2021), 81-102. https://doi.org/10.51302/ tce.2021.614

Guaman, R. E. (2020), EL docente en tiempo de cuarentena, Tecnología educativa docente 8(2), 21-27. https://doi.org/10.37843/rted.v8i2.154

Guisado Salazar, G., Valenzuela Rodríguez, M. A., y Vallejo Quispe, P. A. (2020). Desempeño Docente y el Rendimiento Académico de los Estudiantes de la Facultad de Tecnología en la Universidad Nacional de Educacion de Perú. Revista Conrado, 16(72), 200-203. http://scielo.sld.cu/ scielo.php? script $=$ sci_abstract\&pid $=$ S199086442020000100200\&lng=es\&nrm=iso

Herrera Garzón, V. A. (2021). Desempeño docente en Educación Inicial del circuito "Distrito de Latacunga [Universidad Técnica de Cotopaxi]. In Universidad Técnica De Cotopaxi (Vol. 1). http:// repositorio.utc.edu.ec/bitstream/27000/4501/1/ PI-000727.pdf

Hurtado, F. J. (2020). La educación en tiempos de pandemia, los desafíos de la escuela del siglo XXI.CIEG 44, 176-187. http://www.grupocieg. org/archivos_revista/

Lima Jardilino, J. R., Mendes Sampaio, A. M., y Resende Oliveri, A. M. (2021). Avaliação de desempenho docente: culpar, punir ou desenvolver profissionalmente? Ensaio: Avaliação e Políticas Públicas Em Educação,
29(111), 318-337. https://doi.org/10.1590/ s0104-40362021002902701

Maguiña Polanco, C. M. (2021). Competencias digitales en el desempeño docente en una institución educativa del distrito de San Juan de Miraflores, 2020 [Universidad César Vallejo]. In Universidad César Vallejo (Issue October 2013). http://repositorio.uncp.edu.pe/ bitstream/handle/UNCP/3000/Silva Acosta. pdf? sequence $=1 \&$ is Allowed $=y \% 0$ Ahttps: $/ /$ re positorio.comillas.edu/x m lui / handle/11531/1046

Márquez, J. E. (2017). Tecnologías emergentes, reto parala educación superior colombiana. Ingeniare 23, 35-57. https://doi.org/10.18041/1909-2458/ ingeniare.2.2882

Martínez Chairez, G. I., Esparza Chávez, A. Y., y Gómez Castillo, R. I. (2020). El desempeño docente desde la perspectiva de la práctica profesional. RIDE Revista Iberoamericana Para La Investigación y El Desarrollo Educativo, 11(21). https://doi.org/10.23913/ride.v11i21.703

Mejía Sánchez, Y., y Borgues Oquendo, L. de la C. (2021). Consideraciones para la definición de desempeño profesional en el proceso de calidad en salud. Humanidades Médicas, 21(1), 224238. http://scielo.sld.cu/scielo.php?script=sci_ arttext\&pid=S1727-81202021000100224

Meza Castañeda, A. J. (2021). Acompañamiento pedagógico y desempeño docente en los colegios del distrito de Canta, Lima, 2020 [Universidad César Vallejo]. In Universidad César Vallejo (Issue October 2013). http://repositorio.uncp.edu.pe/ bitstream/handle/UNCP/3000/Silva Acosta. pdf? sequence $=1$ \&is Allowed $=y \% 0$ Ahttps: $/ /$ repositorio.comillas.edu/x mlui/ handle/11531/1046

Ministerio de educación (2016). Propuesta diseño curricular básico nacional para la carrera profesional de profesor de educación inicial. Dirección de Educación Superior Pedagógica Área de Formación Inicial Docente. Lima Perú.

Montilla, R. (2010). Diagnóstico del desempeño docente en educación intercultural bilingüe, caso E.I.B "Zaruma” San Felix-Edo. Bolívar. 
Tesis Licenciatura. Universidad Central de Venezuela. Bolivar.

More Espinoza, R., y Morey Guevara, M. O. (2021). Gestión del clima institucional y desempeño del personal docente de la Institución Educativa $\mathrm{N}^{\circ}$ 7044 San Martín de Porres, Chorrillos. Revista Dilemas Contemporáneas: Educación, Política $y$ Valores, 7044, 6. http://www.scielo.org.mx/ scielo.php?script $=$ sci_arttext\&pid $=$ S2007 $78902021000400006 \& \operatorname{lng}=$ es $\&$ nrm $=$ iso \&tlng $=$ es

Pérez, G. (2000). "La Teoría del Aprendizaje". Recuperado 18 de Mayo 2021 de http://www. jlgcue.es/aprendizaje.htm/

Quispe Malaver, G. E. (2020). Inteligencia emocional y el desempeño docente en las clases virtuales de los profesores de la Red 22, Los Olivos, 2020 [Universidad César Vallejo]. In Universidad César Vallejo (Issue October 2013). http://repositorio.uncp.edu.pe/ bitstream/handle/UNCP/3000/Silva Acosta. pdf? sequence $=1$ \&isAllowed $=y \% 0$ Ahttps: $/ /$ repositorio.comillas .edu/x mlui/ handle/11531/1046

Rodríguez-Marulanda, K. P., y Lechuga-Cardozo, J. I. (2019). Desempeño laboral de los docentes de la Institución Universitaria ITSA. Revista Escuela de Administración de Negocios, 87, 79-101. https://doi.org/10.21158/01208160. n87.2019.2452

Ruiz acuña, W. (2020). Monitoreo docente y práctica pedagógica en la Institución Educativa La Merced de Lima de San Juan de
Miraflores 2020 [Universidad César Vallejo]. In Universidad César Vallejo (Issue October 2013). http://repositorio.uncp.edu.pe/ bitstream/handle/UNCP/3000/Silva Acosta. pdf? sequence $=1$ \&is Allowed $=y \% 0$ Ahttps: $/ /$ repositorio.comillas.edu/x mlui/ handle/11531/1046

Tejedor, S., Cervi, L., Tusa, F., y Parola, A. (2020), Educación en tiempos de pandemia: Reflexiones de alumnos y estudiantes sobre la enseñanza virtual universitaria en España, Italia y Ecuador. Revista Latina de Comunicación Social 78, 1-21. recuperado de https://dialnet.unirioja.es/ servlet/articulo? codigo $=7625686$

UNESCO. Coalición Mundial para la Educación COVID-19. [Internet]. 2020 [citado 12 de mayo de 2021] Disponible en: https://es.unesco.org/ covid19/globaleducationcoalition

Verde Gonzales, H. (2021). Clima organizacional y desempeño laboral en colaboradores de la Unidad de Gestión Educativa Local Tocache, 2020 [Universidad César Vallejo]. In Universidad César Vallejo (Issue October 2013). http://repositorio.uncp.edu.pe/ bitstream/handle/UNCP/3000/Silva Acosta. pdf? sequence $=1 \&$ is Allowed $=y \% 0$ Ahttps: $/ /$ repositorio.comillas.edu/x mlui/ handle/11531/1046 